Classification

Physics Abstracts

$79.60-61.14-71.20-78.70 \mathrm{D}$

\title{
EELS as a fingerprint of the chemical co-ordination of light elements
}

\author{
Rik Brydson $\left({ }^{1,2}\right)$, Hermann Sauer $\left({ }^{1}\right)$, Wilfried Engel $\left({ }^{1}\right)$ and Elmar Zeitler $\left({ }^{1}\right)$. \\ ( $\left.{ }^{1}\right)$ Fritz-Haber-Institut der MPG, Faradayweg 4-6, D1000 Berlin 33, Germany \\ ( $\left.{ }^{2}\right)$ The Blackett Laboratory, Imperial College, London SW7 2BZ, G.B.
}

(Received August 20, 1990; accepted January 28, 1991)

\begin{abstract}
Measurement and modelling of the electron energy loss near-edge structure associated with a particular core-loss edge allows, in favourable cases, the identification of a gross structure reflecting the nearest neighbour environment of the atom undergoing excitation. We present a methodology for the investigation and cataloguing of such co-ordination fingerprints and indicate relevant applications of the technique in both a qualitative and semi-quantitative manner.
\end{abstract}

\section{Introduction.}

In the study of solid state samples, electron energy loss spectroscopy (EELS) constitutes a valuable method of quantitative chemical analysis [1]. The microanalytical results may be directly compared with those obtained from energy dispersive X-ray (EDX) analysis and often provide the sole means of light element analysis. However, additional chemical and structural information may be gained from the measurement and modelling of the electron energy loss near-edge structure (ELNES) associated with each core-loss edge. In many cases it is found that the observed ELNES exhibits a structure specific to the arrangement and type of atoms in the first co-ordination sphere - a so called co-ordination fingerprint $[2,3]$. This essentially arises when the available unoccupied density of states (DOS) is dominated by the interaction of the central atom with the nearest neighbours. A catalogue of such fingerprints may be built up by the investigation of a range of reference materials using EELS together with a comparison with the results obtained using the complementary technique of X-ray absorption spectroscopy (XAS). Further understanding may be derived from the results of a variety of theoretical electronic structure calculations, most notably molecular orbital and multiple scattering calculations. This approach then permits the rapid identification of unknown phases in samples suitable for EELS analysis. 


\section{Discussion.}

The reasons for the existence of co-ordination fingerprints may be understood by consideration of the following points. In complex structures with large, complicated unit cells, there exists very little order outside the first co-ordination shell. This lack of long and medium range order essentially means that the resultant ELNES is dominated by scattering from the nearest neighbour atoms. Furthermore, certain atoms/ions are relatively strong backscatterers, most notably the $\mathrm{O}^{2-}$ ion, and where such species occur in the first co-ordination shell then this will give rise to a potential cage or barrier for electron scattering [4]. This interpretation explains the existence of cation coordination fingerprints in oxides. Another consideration involves the effect of the unscreened core hole potential produced during the excitation process. In certain cases, especially in insulators where the screening is considerably less, the effect of the core hole potential will be to relax the energy levels of available final states to lower energies especially near the edge-onset. This in turn causes the resultant ELNES in this region to be dominated by transitions to bound states essentially formed from the interaction of the central atom with the nearest neighbours [5]. In other words we have a sort of molecular orbital (MO) picture for the resultant ELNES.

ELNES co-ordination fingerprints may be determined by the comparison of EELS spectra obtained from reference compounds with known structural and chemical properties. Minerals constitute a good class of diverse materials: they have usually been well characterized and in most cases they are sufficiently resistant to electron -beam-induced damage as to allow high resolution, low noise measurements (recorded in parallel) to be made. Alternatively ELNES measurements (made with a small collection semi-angle so as to favour dipolar transitions) may be directly compared with XAS measurements, including those made on gaseous or solution phase species where the gross contribution from the nearest neighbour shell is clearly apparent. Measurements on amorphous materials also provide an indication of the contribution from the nearest neighbour shell where the lack of long range order wil minimize the scattering from second and higher neighbour shells.

Interpretation of ELNES fingerprints is best facilitated via comparison with the results of theoretical electronic structure calculations. By far the simplest and most illuminating approach is the assignment of the various near-edge features to transitions to specific molecular orbitals formed from the interaction of atomic orbitals on the central atom in the polyhedral cluster with those on the nearest neighbour atoms. The quantitative MO calculations of Tossell et al. [6-8] are particularly useful in this respect. The nature of the final state MOs are essentially governed by the symmetry of the polyhedral cluster and the number of valence electrons [9]. It therefore follows that isoelectronic species which possess the same symmetry exhibit very similar ELNES (e.g. the $\mathrm{P} / \mathrm{S} / \mathrm{Cl} \mathrm{L}_{2,3}$-edges of $\mathrm{PO}_{4}^{3-}, \mathrm{SO}_{4}^{2-}$ and $\mathrm{ClO}_{4}^{-}$). An alternative approach is to employ the results of multiple scattering calculations performed using the ICXANES computer code of Vvedensky et al. [10]. This method allows the cluster size (number of nearest neighbour shells) to be progressively increased and consequently it is possible to identify the specific scatterers and scattering paths which give rise to various near-edge features. While formally equivalent to a Korringa- KohnRostocker (KKR) band structure calculation, this approach is considerably more flexible allowing the effect of the core hole potential to be included by use of various approximations for the central atom $[11,12]$. The scattering properties of the atoms are described by phase shifts which are calculated by imposing a muffin tin potential on the structure in question and problems may arise in covalent systems which possess directional bonding. Finally, it is possible to employ the projected DOS derived from band structure calculations to describe the origin of the features present in an ELNES fingerprint. However, such an approach requires considerable computational effort in order to determine common electronic structure patterns for related systems. 


\section{Review of ELNES fingerprints.}

In this section we presents a brief review of the co-ordination fingerprints accessible to EELS together with a number of illustrative examples. These have been determined by both EELS and XAS and, while this covers many of the commonly found co-ordinations of light elements, this is not intended to be an exhaustive survey.

\section{Cation fingerprints.}

A good example of a cation fingerprint for two different co-ordinations of the same element are the boron K-ELNES of boron trigonally and tetrahedrally co-ordinated to oxygen $\left(\mathrm{BO}_{3}^{3-}\right.$ and $\mathrm{BO}_{4}^{5-}$ groups) [13]. These are shown in figures $1 \mathrm{a}$ and $2 \mathrm{a}$ respectively and have been measured from the minerals vonsenite and rhodizite.

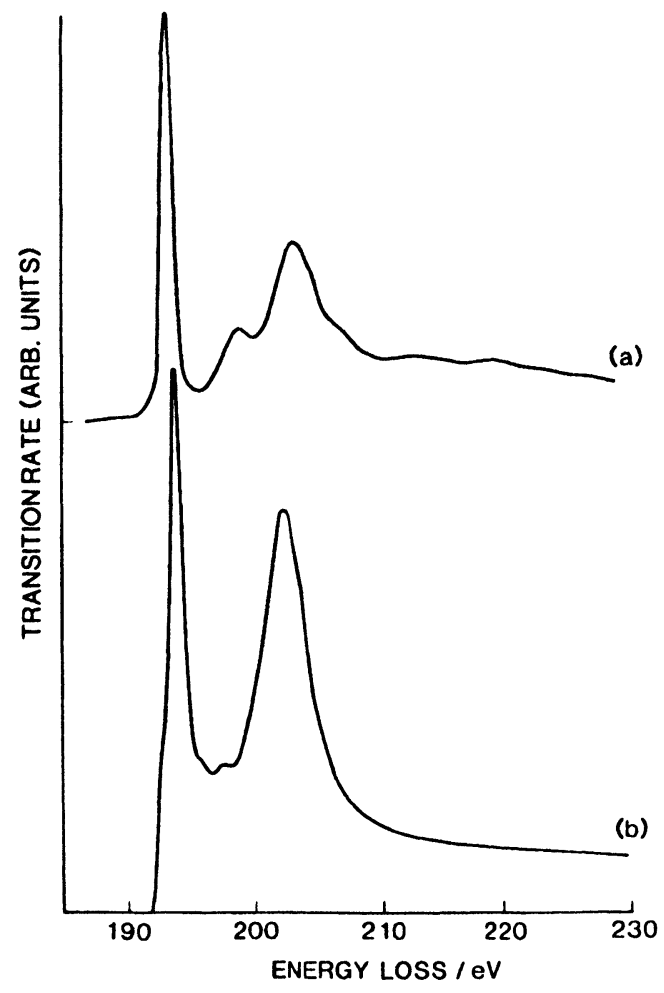

Fig. 1. - (a) Boron K-ELNES of the mineral vonsenite after background subtraction. (b) The results of ICXANES calculations for a single shell of oxygen atoms trigonally co-ordinated to a central boron atom, core hole effects have been included.

The differences arise due to the way the MOs containing boron $2 \mathrm{p}$ character are constructed in the two differing symmetries. In perfect tetrahedral symmetry they remain energetically degenerate as may be seen in figure $2 \mathrm{a}$, however, in planar trigonal symmetry they separate into a $\pi^{*}$ MO and two $\sigma^{*}$ MOs [7]. In figure 1a the transition to the $\pi^{*} \mathrm{MO}$ is evident as a sharp peak at ca. 


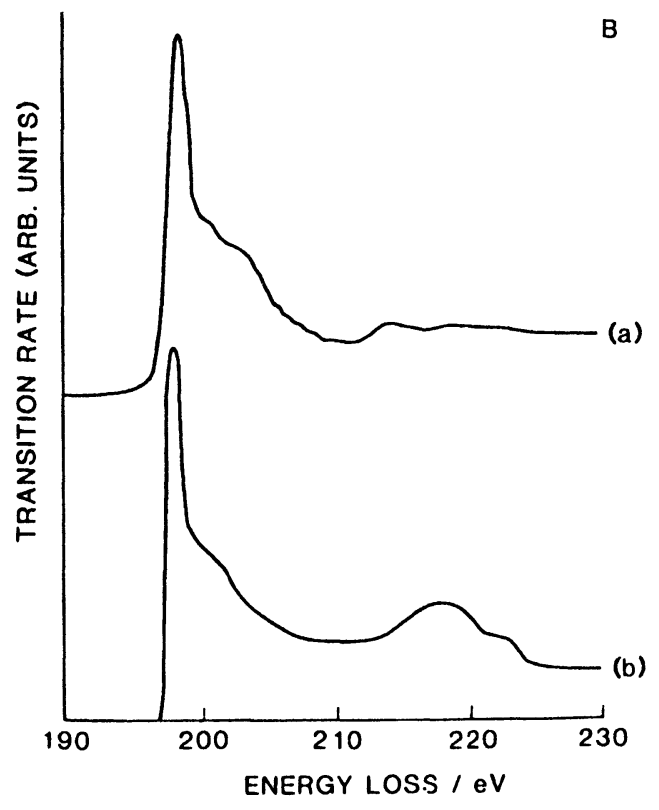

Fig. 2. - (a) Boron K-ELNES of the mineral rhodizite after background subtraction. (b) The results of ICXANES calculations for a single shell of oxygen atoms tetrahedrally co-ordinated to a central boron atom, core hole effects have been included.

$194 \mathrm{eV}$, while the transitions to $\sigma^{*}$ MOs occur higher in energy. The postulate that these spectra are representative of $\mathrm{BO}_{3}$ and $\mathrm{BO}_{4}$ units is confirmed by the results of multiple scattering calculations for $\mathrm{BO}_{3}$ and $\mathrm{BO}_{4}$ clusters shown in figures $1 \mathrm{~b}$ and $2 \mathrm{~b}$. Furthermore, for each co-ordination, the general shape is observed in other compounds where the co-ordination is known to be similar. Since the $\mathrm{BF}_{3}$ molecule is isoelectronic with the $\mathrm{BO}_{3}^{3-}$ ion, the $\mathrm{B}$ K-XANES of gaseous $\mathrm{BF}_{3}$ has the same form as figure 1a [14]. The B K-ELNES of iron boride, FeB has a similar $\pi^{*} / \sigma^{*}$ structure owing to the fact that the co-ordination of boron is trigonal prismatic [15], however, in this case it is considerably broadened and shifted to lower energy which allows the two compounds to be easily distinguished.

Tafto and Zhu [16] have studied the K-ELNES of Mg. Si and Al in both octahedral and tetrahedral co-ordination to oxygen. They observed that the ELNES exhibited a more prominent, wider peak at the edge in the case of octahedral as opposed to tetrahedral co-ordination. We have also found this effect in calculations of the lithium K-ELNES, and seems to arise due to the increased number of nearest neighbour scatterers. Hofer [17] has measured the $\mathrm{Si}_{2,3}$-ELNES of a number of silicates and has identified a fingerprint for the $\mathrm{SiO}_{4}$ tetrahedral unit. Waychunas et al. [18] have investigated the aluminium K-edge from a variety of compounds using XAS. They concluded that the Al K-XANES of tetrahedral aluminium exhibits a peak at $1566 \mathrm{eV}$, while octahedral aluminium displays prominent peaks at 1568 and $1572 \mathrm{eV}$. We have confirmed the octahedral co-ordination of aluminium in the mineral rhodizite via comparison of the observed $\mathrm{Al} \mathrm{L}_{2,3}$ and K-ELNES with those measured from the mineral chrysoberyl (where the Al co-ordination is known to be octahedral) together with the results of multiple scattering calculations for $\mathrm{AlO}_{6}$ and $\mathrm{AlO}_{4}$ clusters $[3,19]$. The results are presented in figures 3 and 4 and clearly show the existence of an aluminium co-ordination fingerprint.

In the case of octahedral co-ordination, we observed that transitions to s-like final states were 


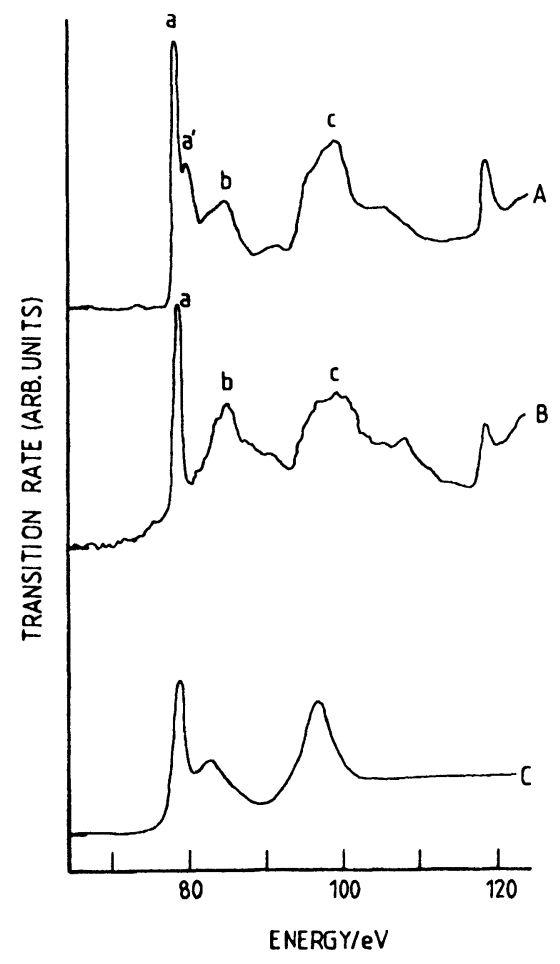

Fig. 3. - Aluminium $\mathrm{L}_{2,3}$-ELNES from (A) chrysoberyl and (B) rhodizite after background subtraction. (C) The results of ICXANES calculations for a single shell of oxygen atoms octahedrally co-ordinated to a central aluminium.

predominant in the region near the edge-onset. Conversely, for the tetrahedral case, transitions to d-like final states dominated this region. This is in agreement with MO calculations [6].

Rhodizite also possesses beryllium in tetrahedral co-ordination to oxygen. Figure 5 shows the Be K-ELNES of rhodizite compared with that of chrysoberyl and with the results of multiple scattering calculations for a $\mathrm{BeO}_{4}$ tetrahedral unit [3,12]. It is apparent the observed structure is characteristic of beryllium tetrahedrally co-ordinated to oxygen.

In the case of the $3 \mathrm{~d}$ transition metals the accessible edges of interest are the $\mathrm{L}_{2,3}$-edges, although much work has been done on the high energy K-edges using XAS [20,21]. Simplistically, the $\mathrm{L}_{2,3}$-ELNES reflect the crystal field splitting of the metal $3 \mathrm{~d}$ band [22] and this is considerably different in octahedral as opposed to tetrahedral co-ordination [23]. In figure 6 we show the titanium $\mathrm{L}_{2,3}$-ELNES for $\mathrm{Ba}_{2} \mathrm{TiO}_{4}$ (tetrahedral Ti) and $\mathrm{BaTiO}_{3}$ (octahedral Ti). These are distinctly different and offer a means of distinguishing between the two co-ordinations [17,24], in principle this should be readily extendable to other $3 d$ transition metal $L_{2,3}$-edges.

\section{Anion fingerprints.}

Carbon exhibits a range of co-ordination fingerprints. It has long been noted that the two allotropes of carbon: graphite and diamond may be distinguished from their C K-ELNES. The presence of $\mathrm{sp}^{2}$ bonding in graphite results in a $\pi^{*}$ peak at $285 \mathrm{eV}$ at the $\mathrm{C} \mathrm{K}$-edge, whereas this is absent in the case of $\mathrm{sp}^{3}$-bonded carbon atoms [25]. Different carbides also show pronounced 

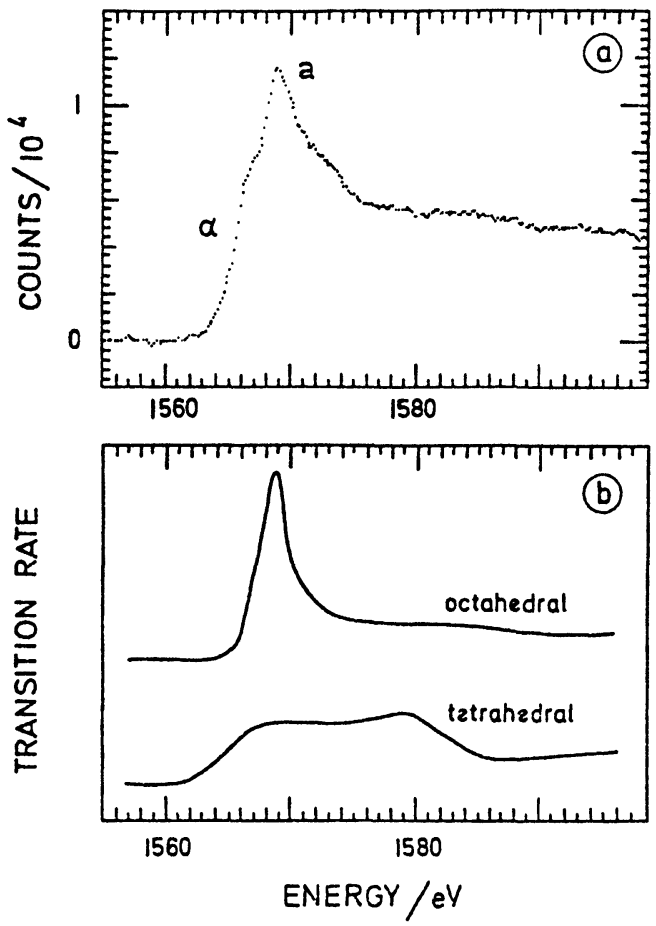

Fig. 4. - (a) Aluminium K-ELNES from rhodizite after background subtraction. (b) The results of ICXANES calculations for aluminium octahedrally and tetrahedrally co-ordinated to oxygen neighbours.

differences [26]. Those containing the acetylide ion, $\mathrm{C}_{2}^{2-}$ show a $\pi^{*} / \sigma^{*}$ structure shown in figure 7 while those possessing isolated $C$ atoms exhibit no such $\pi^{*}$ peak [27]. The $C_{2}^{2-}$ ion is isoelectronic with the molecule $\mathrm{N}_{2}$, and the N K-ELNES of gaseous $\mathrm{N}_{2}$ has a similar form to that in figure 7 [28].

The planar trigonal $\mathrm{CO}_{3}^{2-}$ anion is isoelectronic with the $\mathrm{BO}_{3}^{3-}$ anion and the $\mathrm{C} \mathrm{K}$-ELNES of carbonates all show a similar $\pi^{*} / \sigma^{*}$ structure to that in figure 1a [2]. This can not be confused with the fingerprint for the $\mathrm{C}_{2}^{2-}$ anion since the latter occure some $11 \mathrm{eV}$ lower in energy owing to the less positive effective charge on the carbon atom [26]. Another isoelectronic polyanion with the same $\mathrm{D}_{3 \mathrm{~h}}$ symmetry is the nitrate, $\mathrm{NO}_{3}^{-}$, anion and Hofer [17] has shown that the N K-ELNES co-ordination fingerprint of this species has the same $\pi^{*} / \sigma^{*}$ form.

Hofer has also identified two other N K-ELNES fingerprints [17]. The linear $\mathrm{OCN}^{-}$ion exhibits a familiar $\pi^{*} / \sigma^{*}$ structure, whereas the azide ion $\mathrm{N}_{3}^{-}$which is isoelectronic with $\mathrm{OCN}^{-}$possesses two chemically inequivalent sites owing to the different effective charges on the central and outer nitrogen atoms. The N K-ELNES of $\mathrm{N}_{3}^{-}$therefore consists of two such $\pi^{*} / \sigma^{*}$ structures separated by about $5 \mathrm{eV}$.

The isoelectronic series of polyanions $\mathrm{PO}_{4}^{3-} / \mathrm{SO}_{4}^{2-} / \mathrm{ClO}_{4}^{-}$all exhibit tetrahedral symmetry. The $\mathrm{L}_{2,3}-$ ELNES of phosphorous/ sulphur/ chlorine all show a characteristic twin peaked structure separated by about $10 \mathrm{eV}$ followed at higher energy loss by a much broader peak [29]. This may be easily distinguished from the corresponding ELNES of the element or the phosphide/sulphide/chloride which displays a much broader, featureless near-edge structure [2]. XAS measurements have been performed on the isoelectronic series $\mathrm{SF}_{6}, \mathrm{PF}_{6}^{-}$and $\mathrm{SiF}_{6}^{2-}$ all of which possess 


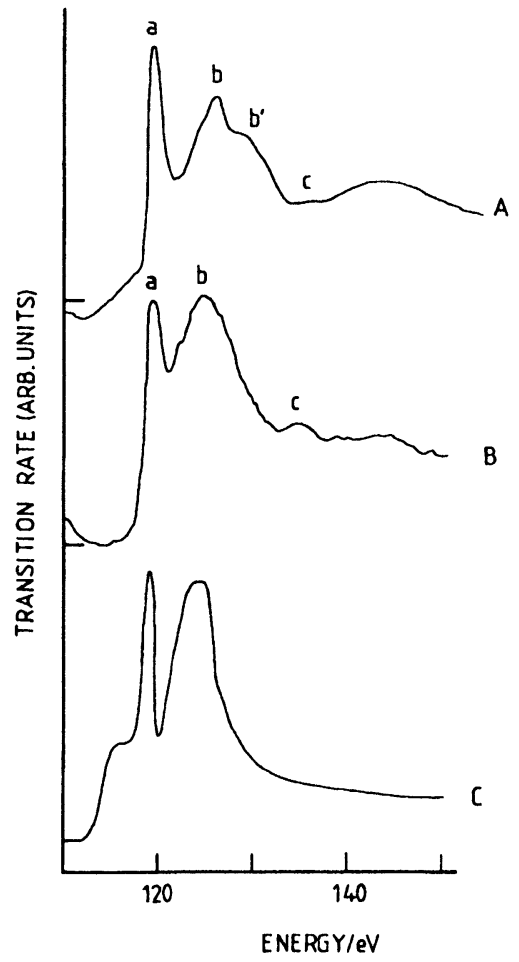

Fig. 5. - Beryllium K-ELNES from (A) chrysoberyl and (B) rhodizite after background subtraction.

(b) The results of ICXANES calculations for aluminium octahedrally co-ordinated to oxygen neighbours.

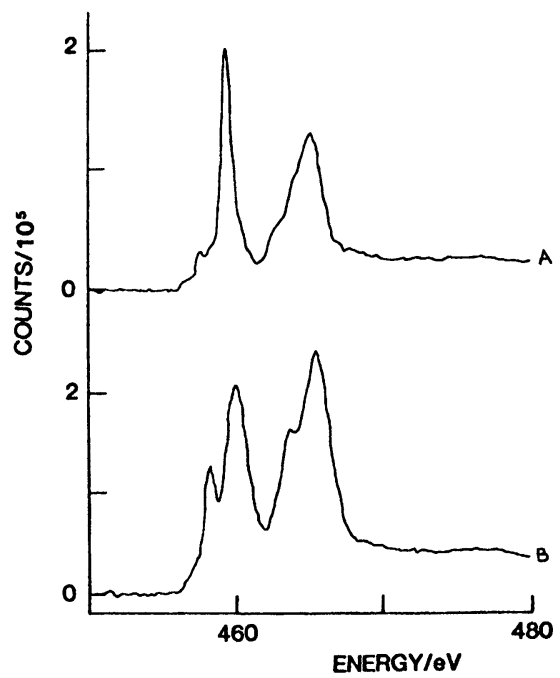

Fig. 6. - Titanium $\mathrm{L}_{2,3}$-ELNES from (a) $\mathrm{Ba}_{2} \mathrm{TiO}_{4}$ and (b) $\mathrm{BaTiO}_{3}$ after background subtraction. 


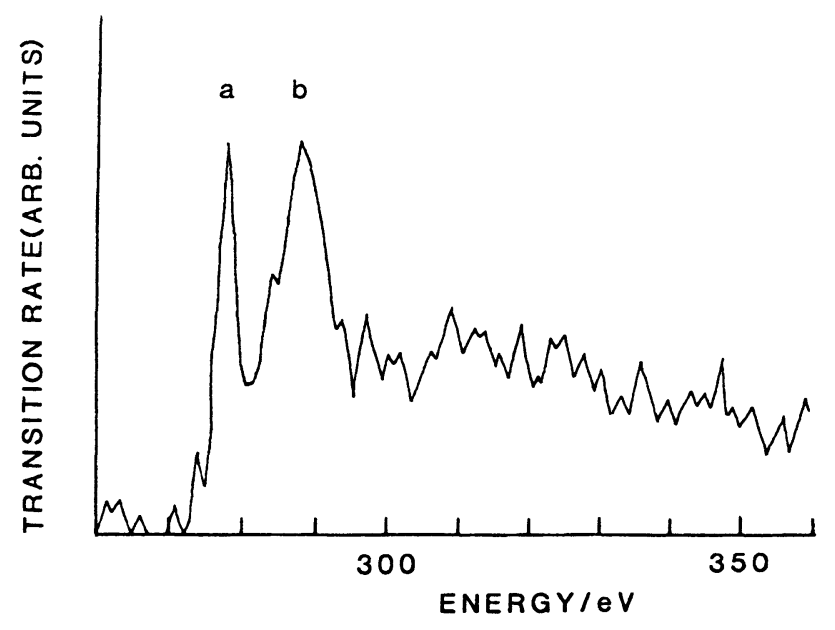

Fig. 7. - Carbon K-ELNES from Li2C2 after background subtraction. The poor statistics are due to the radiation sensiticity of the material.

octahedral symmetry [30]. The $\mathrm{L}_{2,3}$-XANES of sulphur/ phosphorous/ silicon all show a common gross structure which is considerably detailed but can be assigned to transitions to the various MOs of the cluster .

We have performed measurements on the $\mathrm{O} K$-edges of a range of titanium (IV) oxides [31]. In all cases oxygen is co-ordinated to titanium and the first five $\mathrm{eV}$ of the $\mathrm{O} \mathrm{K}$-ELNES is dominated by transitions to $\mathrm{O} 2 \mathrm{p}-\mathrm{Ti} 3 \mathrm{~d}$ hybridized orbitals. This leads to a twin peaked structure which reflects transitions to $\mathrm{p} \pi$ and $\mathrm{p} \sigma$ antibonding MOs formed from the interaction of oxygen with the titanium nearest neighbours. The relative intensities of these two peaks vary with changes in the co-ordination symmetry, and in the case of cubic perovskites there appears to be a common edge structure due to the linear, twofold co-ordination of titanium to oxygen. To illustrate this, figures 8 and 9 show the $\mathrm{O}$ K-ELNES of two cubic perovskites, $\mathrm{BaTiO}_{3}$ and $\mathrm{SrTiO}_{3}$ together with the results of multiple scattering calculations. In order to reproduce all the observed structure at least seven shells are required. However, the first five $\mathrm{eV}$ of the observed ELNES may be adequately modelled using only the nearest neighbour shell, indicating the existence of a co-ordination fingerprint.

\section{Further applications.}

When the ELNES fingerprints of two different co-ordinations of the same element have been determined, it is often possible to apply this to compounds where both co-ordinations co-exist and so determine the relative site occupancies via an algorithm or fitting procedure. Berger et al. [32] have applied this technique to the determination of the proportion of $\mathrm{sp}^{2-}$ bonded carbon atoms in amorphous diamond films. Since $\mathrm{sp}^{2}$-bonded carbon gives rise to a $\pi^{*}$ peak in the $\mathrm{C}$ K-ELNES, measurement of the relative area of this peak gives a measure of the contribution of this species to the total ELNES. We have applied a similar algorithm in order to determine the proportion of $\mathrm{BO}_{3}$ and $\mathrm{BO}_{4}$ groups in various boron-oxygen compounds. The validity of the method was confirmed by performing the analysis on various boron minerals where the structure, and hence the relative site occupancy, is known [13]. The technique was then applied to determine the proportion of $\mathrm{BO}_{3}$ groups in boron-doped oxide films formed on an $\mathrm{Fe}-\mathrm{Cr}$ alloy [15]. This 


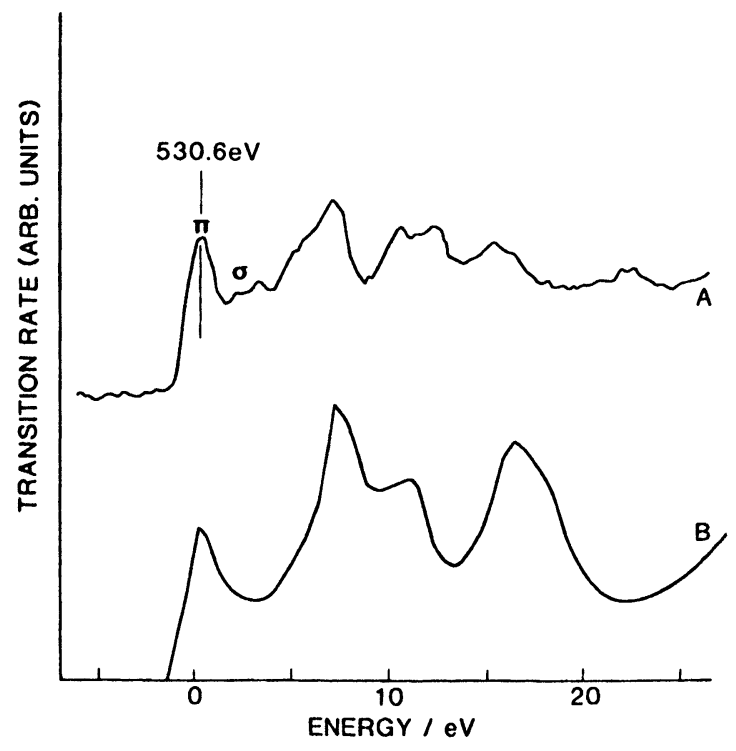

Fig. 8. - (A) Oxygen K-ELNES from $\mathrm{BaTiO}_{3}$ after background subtraction. (B) The results of ICXANES calculations for the $\mathrm{O}$ K-edge in $\mathrm{BaTiO}_{3}$, seven shells have been included.

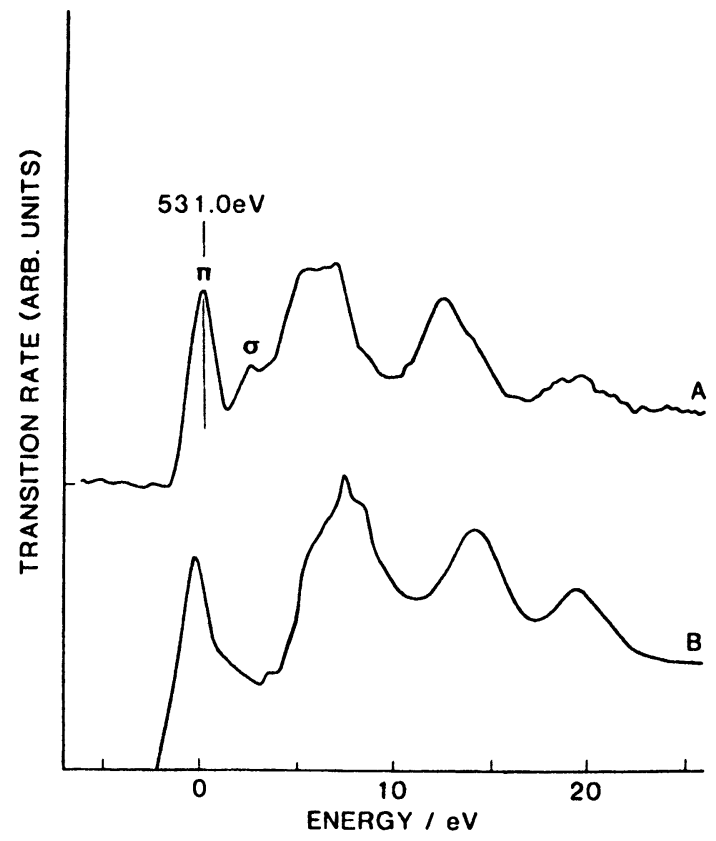

Fig. 9. - (A) Oxygen K-ELNES from $\mathrm{SrTiO}_{3}$ after background subtraction. (B) The results of ICXANES calculations for the $\mathrm{O}$ K-edge in $\mathrm{SrTiO}_{3}$, seven shells have been included. 
information then allowed us to postulate various mechanisms for the oxidation behaviour of such systems.

Small variations in co-ordination fingerprints can often be related to differing degrees of distortion from perfect co-ordination (i.e. variations in bond lengths and bond angles) [33]. Comparison of experimentally observed spectra with those derived from standards together with the results of various trial calculations often yield a measure of the amount of distortion. Measurements of the Ti L 2,3-ELNES from a series of titanium (IV) oxides, chosen so that the tetragonal distortion in the Ti co-ordination octahedron gradually increased, indicated that the broadening and splitting of the near-edge features increased as the Ti site symmetry was lowered [34]. Bianconi et al. [35] have postulated a general rule which states that the energy of a scattering shape resonance ( $\sigma^{*}$ resonance) above the edge onset is proportional to $1 / R^{2}$, where $R$ is the bond length. Identification of such shape resonances, which often appear as broad peaks above the intial fine structure corresponding to bound-state transitions, permits a semi-quantitative determination of the corresponding bond-lengths.

\section{Conclusion.}

In this article we have attempted to show how a systematic study of ELNES measured from a series of compounds is required if a catalogue of co-ordination fingerprints is to be built up. The formation of such a reference work would aid routine microanalysis, allowing the rapid identification of unknown phases in complex systems. We have also indicated further applications of ELNES fingerprints, including their use as a probe of local distortion and as a means of the quantifying relative site occupancies.

\section{Acknowledgements.}

One of us (R.B.) is grateful to the Royal Society for financial support for this research.

\section{References}

[1] Williams B.G., Prog. Solid State Chem. 17 (1987) 87.

[2] HOFER F. and GOLOB P., Ultramicroscopy 21 (1987) 379.

[3] BRydson R., Sauer H., Engel W., ThOMas J.M. and Zertler E., J. Chem. Soc. Chem. Commun. 15 (1989) 1010.

[4] Bianconi A., Dell'ariccia M., Durham P.J. and Pendry J.B., Phys. Rev. B 26 (1982) 6502.

[5] BIANCONI A., in EXAFS and Near-Edge Structures III', K.O. Hodgson, B. Hedman and J.E. PennerHahn Eds. Springer Proc. Phys. 2 (Springer Verlag: New York, 1984).

[6 Tossell J.A., J. Phys. Chem. Solids 36 (1975) 1273; Tossell J.A., J. Am. Chem. Soc. 97 (1975) 4840.

[7] VAughan D.J. and TOSSELl J.A., Am. Mineralogist 58 (1973) 765.

[8] TOSSELl J.A., VAUGHAN D.J. and JOHNSON K.H., Am. Mineralogist 59 (1974) 319.

[9] WALSH A.D., J. Chem. Soc. (1953) 2301.

[10] VVEDENSKy D.D., SALDin D.K. and PENDRY J.B., Computer Phys. Comm. 40 (1986) 421.

[11] Linder Th., SAUER H., ENGEL W. and KAMBE K., Phys. Rev. B 33 (1986) 22

[12] BRYdSON R., VVEDENSKY D.D., ENGEL W., SAUER H., Williams B.G., ZEITLER E. and ThOMAS J.M., J. Phys. Chem. 92 (1988) 962.

[13] Brydson R., SAUER H., Rowley P., Engel W., Thomas J.M. and Zertler E., J. Chem. Phys., to be submitted, SAUER H., BRYdSON R., ENGEL W. and ROWLEY P.N., Proc. XII ICEM, Vol.2, p.54 (S.F. Press: San Francisco 1990). 
[14] HaYes W. and Brown F.C., J. Phys. B 4 (1971) 185.

[15] Rowley P.N., BRydson R., Little J., SAUNDERS S.R.J., SAUER H. and Engel W., Oxid. Met. 35 (1991) 375.

[16] TAFTO J. and ZHU J., Ultramicroscopy 9 (1982) 349.

[17] HOFER F., Habilitationsschrift, Technische Universitat Graz (1988).

[18] WAYChUNAS G.A. and BROWN G.E., in Ref. [5].

[19] Brydson R., Williams B.G., Sauer H., Engel W., Schlogl R., Muller M., Zeitler E. and ThOMAS J.M., J. Chem. Soc. Faraday Trans. 184 (1988) 631.

[20] Lytle F.W., GReEgor R.B. and PANSON A.J., Phys. Rev. B 37 (1988) 1550.

[21] WONG J., LyTLE F.W., MESSMER R.P. and MAYLOTTE D.H., Phys. Rev. B 30 (1984) 5596.

[22] de Groot F.M.F., FugGle J.C., Thole B.T. and SawatzKY G.A., Phys. Rev. B 41 (1990) 928.

[23] BALlhauSEN G.J., Introduction to Ligand Field Theory (McGraw Hill: New York, 1962).

[24] BRYDSON R., THESIS Ph.D., University of Cambridge (1988).

[25] Colliex C., Manoubi T., Gasgnier M. and Brown L.M., Scanning Electron Microsc. 2 (1985) 489.

[26 BRydson R., BRULEY J. and Thomas J.M., Chem. Phys. Lett. 149 (1988) 343.

[27] Disko M.M., SPENCE J.C.H., SANKEY O.F. and SAldin D.K., Phys. Rev. B 33 (1986) 5642.

[28] HITCHCOCK A.P. and BRION C.E., J. Electron Spectrosc. 27 (1980) 243.

[29] Sekiyama H., Kitajima Y., Kogusi N., Kuroda H. and Ohta T., Photon Factory Activity Report 1983/84, VI-119 (1984).

[30] SekiYama H., Kitajima Y., Kogusi N., Kuroda H. and OHTa T., Photon Factory Activity Report 1983/84, VI-120 (1984).

[31] Sauer H., Engel W., Brydson R. and Hofer F., submitted to J. Phys. Cond. Matter.

[32] Berger S.D., McKenZie D.R. and MarTin P.J., Philos. Mag. Lett. 57 (1988) 285.

[33] BufFat B. and Tuilier M.H., Solid State Commun. 64 (1987) 401.

[34] SAUER H., ENGEL W. and HOFER F., Proceedings of Salzburg 89, Dreilanderstagung Elektronen Mikroskopie (10-16 September 1989).

[35] BIANCONI A., DELl'ARICCIA M., GARgaNo A. and NATOli C.R. EXAFS and Near-Edge Structures, A. Bianconi, L. Innocia and S. Stipchich Eds. (Springer Verlag: New York, 1983). 